\title{
Encuesta sobre la seducción y el cortejo a los estudiantes de la Universitat Pompeu Fabra (20 a 27 años)
}

\section{Seduction survey at the university students of the Universitat Pompeu Fabra (from 20 to 27 years old)}

\author{
Carmen Maté $\quad$ Nolasc Acarín \\ Agencia de Ecología Urbana, España \\ (Rec: Julio 2011 - Acep: Octubre 2011)
}

\begin{abstract}
Resumen
El objetivo de este estudio es mostrar si los universitarios son conscientes de las fases de la seducción y si son capaces de identificarlas. Las fases de galanteo y seducción son etológicamente muy interesantes, pero no suelen ser objeto de atención en las encuestas sobre sexualidad. El estudio comprende una muestra de 838 estudiantes universitarios a quienes se les pasó una encuesta con 12 preguntas relacionadas con la seducción. El $70 \%$ reconoce que el inicio se produce mediante el contacto visual y que forma parte de las estrategias de aproximación. Durante el proceso de la seducción, las dos terceras partes de la muestra resaltan la necesidad de establecer una base de confianza. En cuanto a las estrategias para encontrar intereses comunes, ambos sexos prefieren encontrar similitudes en personalidad y en intereses socioculturales. La mitad responde que son los varones quienes inician el contacto. Las respuestas sobre las conductas que emplean para mostrarse de forma favorable muestran diferentes estrategias en la seducción. Respecto al establecimiento de la relación, los hombres responden más frecuentemente que ésta se establece después de mantener relaciones sexuales y las mujeres cuando hay facilidad en la comunicación.
\end{abstract}

Palabras clave: proceso de seducción, diferencias de género, encuesta a universitarios.

\begin{abstract}
This study aims to show whether universitary students are conscious about the seduction phases of seduction and if they are capable to identify them. From the point of the ethological view, courtship and seduction phases are very interesting and they have received less attention in sexuality surveys. The sample of this study was 838 students who answered 12 questions related to the seduction. $70 \%$ recognize startup occurs through eye contact, and this is also a part of the approach strategies. During the process of the seduction, two-thirds of the sample remarked the necessity to establish a confidence base. As far as the strategies to find common interests, both sexes prefer to find personality similarities and common socio-cultural interests. The answers on the behaviors used to show favorable way, once selected and prioritized show different strategies in seduction. The establishment of the relation the men answer more frequently the relationship is established to maintain sex and women when there is ease in communication.
\end{abstract}

Key words: seduction process, gender differences, students survey.

\footnotetext{
Correspondencia a: Carmen Maté. Dirección: c/Escar n¹, 30 08039 Barcelona, España. E-mail: carmenmate@ bcncologia.net.
} 


\section{Introducción}

En este artículo se estudia cuál es el conocimiento que los universitarios tienen del proceso que se desencadena en la seducción. Las preguntas formuladas en la encuesta (12) detallan las diferentes fases del proceso de festejo que se han observado en todas las culturas (Eibl-Eibesfeldt, 1993). El estudio del comportamiento sexual en nuestra especie suele limitarse al acto sexual y a la elección de pareja (Masters \& Jonson, 1966; Buss, 1994; Lauman, Gagnon, Michael, Michaels, 1994 Maté \& Acarín, 2010). En cambio; las fases de la toma de contacto, del galanteo y el enamoramiento, desde la perspectiva etológica mucho más interesantes, han sido objeto de menor atención.

En otras especies se observa cómo aquellas que practican un emparejamiento sexual duradero se unen, por lo general, tras una fase prolongada de cortejo (Wickler \& Seibt, 1977). Una interpretación generalizada es que cuanto mayor es el esfuerzo realizado por el macho, más importante es para él conservar a su pareja para cuadrar el coste-beneficio. Así también cuanto más esquiva sea la hembra, más valiosa aparecerá hasta una proporción óptima.

Nuestro repertorio de estrategias de emparejamiento es amplio y sensible al contexto, la puesta en práctica de determinadas estrategias sexuales del repertorio humano no solo depende de las circunstancias individuales, sino también de las culturales. El contexto lo es todo. Es probable que los contextos recurrentes de nuestro pasado evolutivo favorecieran estrategias que perviven en la actualidad. No obstante, el contexto del momento y las condiciones culturales determinan qué estrategias se activan y cuáles no.

El galanteo, el cortejo o la seducción, son un conjunto de estrategias para superar el temor al contacto, que se realizan en diversas etapas y en las que se pueden identificar varias fases para la aproximación. Lo importante es que ésta se produzca de forma sutil, cuando los interlocutores todavía no se tienen confianza.

El contacto visual es primordial para establecer una relación, cuando se está a una cierta distancia. Con la mirada se busca el contacto y se comunica al interlocutor que se halla en el punto de mira. La blancura de la pupila permite leer la dirección de las miradas y si el interlocutor encuentra réplica, en principio significa una respuesta favorable. Pero el contacto visual no es un compromiso, es solo el inicio. Esta primera fase de la secuencia permite calibrar las cualidades físicas del otro, proporcionando información sobre edad, tipo, color, estatus y algo sobre el carácter que en su conjunto permite evaluar si el otro atrae, o no. Después los intercambios verbales permiten comprobar la predisposición para otros contactos. En esta fase el marco cultural determina las estrategias de acercamiento, también depende del grado de conocimiento previo ya que se esforzaran para establecer un sistema de relación común. Si no hay una tercera persona para las presentaciones, se inicia el intercambio verbal con comentarios triviales. La conversación intrascendente también aporta información más detallada del individuo y el oído completa la información sobre la dicción, el tono de voz, el acento, la fluidez de expresión, la manera de pensar y la utilización del vocabulario (Medina, 1991). En esta fase se comunican los intereses comunes y se expresan las coincidencias con movimientos afirmativos de cabeza.

El establecimiento de la base de confianza consiste en descubrir las debilidades a la pareja pero siempre contrarrestando con una presentación favorable de uno mismo, o lo que se puede denominar una imposición de reclamo (Eibl-Eibesfeldt, 1993) destinada a causar impresión en el contrario. En la autopresentación favorable cuenta tanto el dominio sobre el entorno social como la presentación de uno mismo como proveedor fiable y competente. En todas las culturas, durante el cortejo se recurre a la autopresentación favorable, a requerimientos de asistencia y a demandas infantiles (Eibl-Eibesfefedt, 1971).

El cómo se establece el contacto corporal varía culturalmente, aunque por lo general son los hombres quienes toman la iniciativa al tocar de la pareja de forma aparentemente casual. El varón se ofrece, mientras que la mujer selecciona si acepta o rechaza, esto no excluye que también las mujeres lleven la iniciativa en el proceso del cortejo. Los primeros contactos físicos suelen producirse con una excusa de ayudar, o dar protección corporal o como para guiar en una dirección. Una vez iniciado el contacto, si hay consentimiento al contacto físico, puede pasarse a un grado superior de intimidad mediante un abrazo móvil. Para superar la barrera de la intimidad se utilizan las pautas de los abrazos, caricias y besos, que son las mismas que utilizamos en las relaciones materno-filiales o amistosas, pero en las relaciones sexuales estas muestras de cariño son el preludio al coito. Hasta llegar al contacto íntimo, el cortejo se ritualiza y se formaliza para conocer y establecer confianza con la pareja.

Así, la formación de la pareja se realiza en varias fases cuya sucesión depende del conocimiento previo entre ambos. En la constitución de cualquier relación social, los interlocutores se esfuerzan por conservar su 
propia capacidad de decisión y al mismo tiempo se trata de hacer que los intereses propios sean los de la pareja, de ganarla para las mismas metas. Evidentemente la influencia cultural propicia cambios en la reducción de las secuencias o en la alteración de las pautas de comportamiento o en el cambio de orden de las mismas.

El objetivo de este estudio es mostrar si los universitarios son conscientes de las fases de la seducción y si son capaces de identificarlas. También nos interesa verificar si se producen variaciones en las fases del cortejo.

\section{Método}

\section{Participantes}

La población investigada son los estudiantes de Humanidades de la UPF que tienen una media de edad de 22 años -el mínimo de 19 y el máximo de 41-; el $96,2 \%$ de la muestra está comprendida entre 19 a 27 años. La encuesta se inició en el curso 1999-2000 y finalizó el 2003-2004, participando un total de 838 alumnos (253 varones y 585 mujeres), todos voluntarios y anónimos. El universo fue el conjunto de estudiantes de Humanidades que se matriculaban en las asignaturas impartidas por los autores (240 estudiantes/año), la totalidad de alumnos de los cursos de tercero y cuarto era de 1200 (Maté \& Acarín, 2010, 2011).

\section{Instrumentos}

La encuesta, elaborada por los autores, consistía en 42 preguntas acerca, de sus experiencias y actitudes sexuales. En concreto en este artículo se analizan las doce que estaban relacionadas con el festejo, las otras trataban de aspectos genéricos de las relaciones sexuales y características destacables en la elección de pareja (Maté \& Acarín, 2010, 2011).

\section{Procedimiento}

El error de la muestra, en el supuesto de una selección aleatoria, para un nivel de confianza de $95 \%(2 \sigma)$ es de $0,01(1 \%)$ para el conjunto de la muestra, siendo $\mathrm{N}=1200$, que era la totalidad de alumnos en los cursos de tercero-cuarto, durante el periodo de estudio. Para el análisis confirmatorio se igualaron las muestras de ambos sexos $\mathrm{N}=500$ (250 varones y 250 mujeres), para comprobar si existían diferencias en sus respuestas. La muestra de las mujeres $(\mathrm{n}=585)$ y de los hombres $(n=253)$ se seleccionaron al azar con una opción que tiene el programa de análisis estadístico SPSS.

\section{Análisis de los datos}

Las pruebas estadísticas empleadas para las variables cualitativas son la tablas de contingencia con el cálculo de la $\chi^{2}$ de Pearson y los residuos ajustados -empleando como referencia el valor estadístico de prueba 1,96 en valor absoluto para una distribución normal y un nivel de significación de 0,05- (Haberman, 1978). Cuando en el $80 \%$ de las celdas de las tablas de contingencia los valores esperados eran inferiores a 5, se utilizó la prueba exacta de Fisher.

\section{Resultados}

\section{Estrategias de aproximación}

Preguntados sobre las estrategias de aproximación durante el festejo, el 69,8\% ( $\mathrm{n}=585)$ busca el contacto visual, el 24\% $(n=201)$ hace una aproximación directa y desinhibida, el 4,2\% (n=35) NS/NC (No sabe /No Contesta) y el $2 \%(n=17)$ utiliza a terceras personas. $\mathrm{Al}$ analizar los datos en función del sexo para ver si existen diferencias en las estrategias de aproximación, se obtienen diferencias estadísticamente significativas en las siguientes respuestas: los hombres marcan con mayor frecuencia la respuesta "aproximación directa", en cambio las mujeres el "contacto visual" $\left(\chi^{2}(3)=14,1\right.$ $\mathrm{p}<0,005$ ) (Fig. 1).

Figura 1. Diferencias entre hombres y mujeres en la estrategia de aproximación.

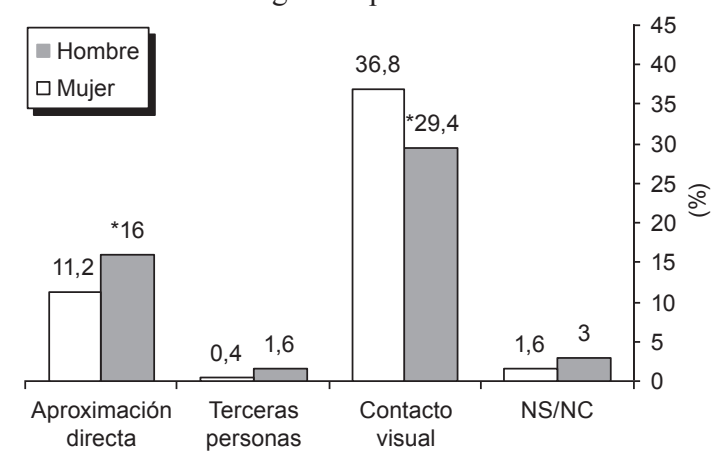

Acerca de la actitud durante el proceso de seducción, el 77,7\% (n=651) establece una base de confian$\mathrm{za}$, el $16,1 \%(\mathrm{n}=135)$ opta por provocar formulando opiniones contrarias, un 3,1\% $(n=26)$ opta por hacer afirmaciones continuamente y un 3,1\% ( $n=26) \mathrm{NS} / \mathrm{NC}$. No se obtienen diferencias estadísticamente significativas al comparar las respuestas en función del sexo, en el proceso de festejo $\left(\chi^{2}(3)=2,83 ; p>0,05\right)$. 
Figura 2. Diferencias entre hombres y mujeres, sobre quién inicia el primer contacto.

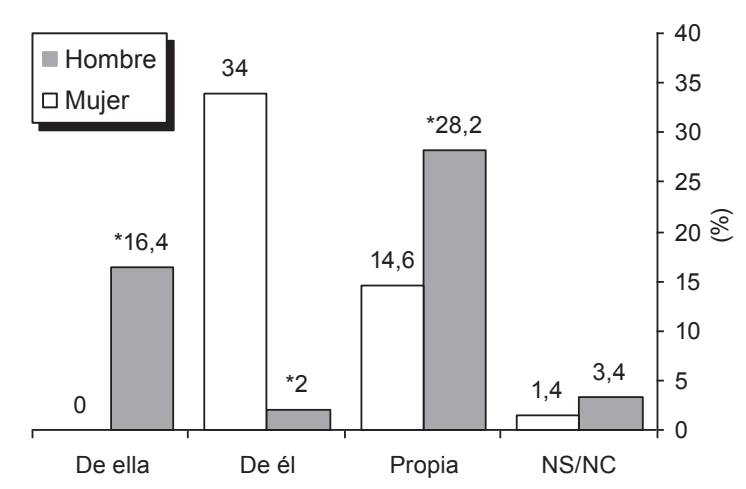

\section{Posición de reclamo}

Las respuestas sobre las conductas que emplean para mostrarse de forma favorable, "posición de reclamo", debían seleccionarlas y priorizarlas. En la respuesta sobre la ostentación de la capacidad de dominio no se obtienen diferencias estadísticamente significativas entre los $\operatorname{sexos}\left(\chi^{2}(3)=4,44 ; p>0,05\right)$, siendo una opción marcada como la primera por el $19,5 \%(n=163)$, como segunda opción la marca el $25,3 \%(\mathrm{n}=212)$ y como tercera un $22 \%(n=184)$. Tampoco se obtienen diferencias en función de los sexos respecto a la estrategia de utilizar "requerimientos infantiles" para provocar una respuesta protectora $\left(\chi^{2}(3)=3,88 ; p>0,05\right)$, pero el $26,6 \%$ marca esta estrategia en segundo lugar. En cambio, sí se obtienen diferencias estadísticamente significativas entre hombres y mujeres respecto a la "presentación como un proveedor fiable", más valorado por las mujeres $\left(\chi^{2}(3)=36,64 ; \mathrm{p}<0,001 * *\right)$ como estrategia de seducción de los hombres. También se obtienen diferencias estadísticamente significativas respecto a la actitud juguetona, siendo los hombres quienes más valoran esta conducta en las mujeres $\left(\chi^{2}\right.$ (3) $=12,51 ; \mathrm{p}<0,05 *)$.

Al tener que priorizar las respuestas sobre los aspectos de la seducción, equiparamos el presentarse de forma favorable con la "posición de reclamo", teniendo en cuenta toda la muestra, la primera opción es "mantener una actitud juguetona" con un 47,9\% ( $\mathrm{n}=401)$, un $28 \%(\mathrm{n}=235)$ marca como la segunda el "provocar una respuesta protectora" y un $13,2 \%(\mathrm{n}=111)$ se presenta como un proveedor fiable.

\section{Iniciativa al contacto}

Cuando se pregunta sobre quién toma la iniciativa en el primer contacto, el 49,4\% ( $n=414)$ marca que son los hombres, solo un $10 \%(\mathrm{n}=84)$ las mujeres, el $36,4 \%$ ( $n=305)$ uno mismo y el 4,2\% $(n=35)$ NS/NC. Se obtienen diferencias estadísticamente significativas al comparar los sexos, siendo los hombres los que inician el primer contacto $\left(\chi^{2}(3)=249,99 ; \mathrm{p}<0,001 * *\right)$ (Fig. 2$)$.

La situación en la que se produce con más frecuencia el contacto corporal, es bailando con un $36,5 \%$ $(\mathrm{n}=306)$, un $30,4 \%(\mathrm{n}=255)$ ayudando a la pareja, $8 \%(n=67)$ ofreciendo algún objeto, $8,5 \%(n=71)$ conversando y un $8,8 \%(n=74)$ otras. No se obtienen diferencias estadísticamente significativas al comparar las respuestas de ambos sexos si el contacto se hace bailando $(\mathrm{F}=1,32 ; \mathrm{p}>0,05)$ o ayudando a la pareja a ponerse el abrigo, o a levantarse $(\mathrm{F}=1,5 ; \mathrm{p}>0,05)$. En cambio, sí se observan diferencias entre los sexos en la respuesta de ofrecer un objeto $\left(\mathrm{F}=9,9 ; \mathrm{p}<0,05^{*}\right)$.

Preguntados sobre cuáles son las conductas que llevan a cabo para mostrar ternura y como preludio sexual, el $41,4 \%(n=347)$ marca los besos en primer lugar, el 43,6\% $(n=365)$ las caricias en segundo lugar, $y$ los abrazos en tercer lugar con $12,1 \%(\mathrm{n}=101)$, mientras que el $3,1 \%(n=25)$ NS/NC. No se obtienen diferencias estadísticamente significativas entre hombres y mujeres en las respuestas: besos $\left(\chi^{2}(3)=7,38 ; p>0,05\right)$, caricias $\left(\chi^{2}(3)=4,32 ; p>0,05\right)$ abrazos $\left(\chi^{2}(3)=3,67 ; p>0,05\right), y$ cosquillas $(F=3,62 ; P>0,05)$. En la estrategia durante el festejo para intentar encontrar intereses comunes, no se obtienen diferencias estadísticamente significativas al comparar las respuestas de hombres y mujeres $(\mathrm{F}=7,87$; $\mathrm{p}>0,05)$. Ambos prefieren encontrar intereses comunes relacionados con los rasgos de personalidad de cada uno con el 57,6\% $(n=483)$, el 29,5\% $(n=247)$ resalta el compartir intereses de tipo sociocultural, un $8,9 \%(n=75)$ el compartir valores éticos, un 2,6\% $(n=22) \mathrm{NS} / \mathrm{NC}$ i $11(1,3 \%)$ otros.

\section{Sobre el festejo y la seducción}

Acerca de la fase del festejo que les parece más importante, el $49,4 \%(n=414)$ marca que es la presentación de uno mismo, el 26,4\% $(n=221)$ aparecer como deseable, un $15,5 \%(n=130)$ es causar buena impresión, un 6,7\% $(\mathrm{n}=56)$ solicitar pequeños favores y un $2 \%(n=17)$ NS/NC. No se observan diferencias estadísticamente significativas entre hombres y mujeres en las respuestas ( $\mathrm{F}=6,87 ; \mathrm{p}>0,05)$. La fase del festejo que consideran como muy importante es la presentación de uno mismo (el porcentaje por sexo es de 51\%).

Sobre si consideran que la seducción y el festejo son imprescindibles el 77,2\% $(n=647)$ responde que sí, frente a un 10,9\% (n=91) que dice que no. Se obtienen diferencias estadísticamente significativas 
entre los hombres y las mujeres al considerar imprescindible la seducción $\left(\mathrm{F}=9,71 ; \mathrm{p}<0.05^{*}\right)$, siendo las mujeres quienes más responden afirmativamente y los hombres los que más de forma negativa (Fig. 3).

Figura 3. Las diferencias entre hombres y mujeres respecto a si consideran indispensable la seducción.

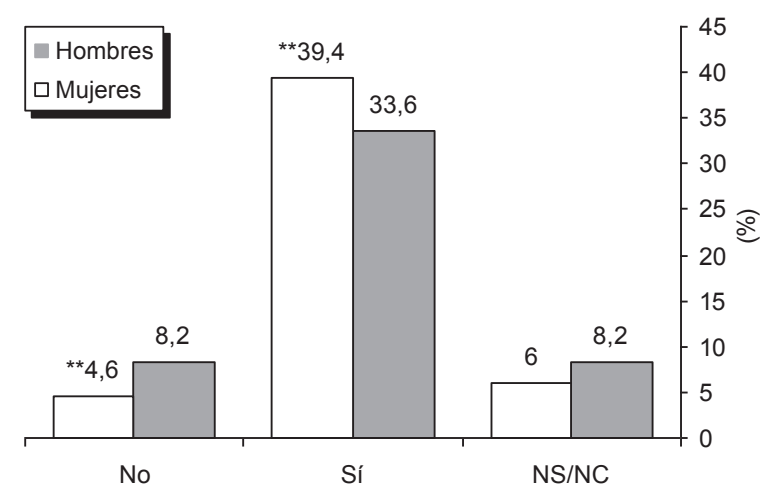

Visión sobre la relación de pareja

El 38,3\% (n=321) de los universitarios encuestados considera que ha establecido una relación de pareja cuando se manifiesta una estimación mutua, el 5,7\% $(\mathrm{n}=48)$ cuando hay facilidad en la comunicación, un $1,8 \%(\mathrm{n}=15)$ después de haber mantenido relaciones sexuales y el $46,1 \%(n=386)$ cuando se dan todos los supuestos anteriores. Se obtienen diferencias estadísticamente significativas en las respuestas de ambos sexos, los hombres responden más frecuentemente que la relación se establece después de mantener relaciones sexuales y las mujeres cuando hay facilidad en la comunicación $\left(\chi^{2}(5)=23,33 ; p<0,001 * *\right)$. Para saber qué piensan sobre cómo evoluciona la relación de pareja se proponen cinco posibles fases que tienen que seleccionar y priorizar. El 88,3\% ( $\mathrm{n}=740)$ marca primero el establecimiento de confianza, en segundo lugar con $62,5 \%(\mathrm{n}=524)$ el tener un proyecto en común, la tercera con $46,7 \%(\mathrm{n}=391)$ la repartición de roles, la cuarta con 39,7\% $(\mathrm{n}=333)$ la atención a los hijos y la quinta con $42,7 \%(n=358)$ el interés patrimonial. Solo se obtienen diferencias estadísticamente significativas entre hombres y mujeres en las respuestas de establecer un proyecto en común $\left(\chi^{2}(5)=9,57 ; p<0,05^{*}\right)$ y en la del interés patrimonial $\left(\mathrm{F}=18,88 \mathrm{p}<0,05^{*}\right)$, que son más valoradas por ellas. Sobre la visión que tienen sobre la pareja, el 55,3\% $(n=463)$ marca la opción romántica, un $29,4 \%(n=246)$ la define como realista, un $8,5 \%$ $(\mathrm{n}=70)$ como práctica, y un $5,7 \%(\mathrm{n}=48)$ directa.
Se obtienen diferencias estadísticamente significativas entre hombres y mujeres en la visión que tienen de la relación de pareja $(\mathrm{F}=25,8 ; \mathrm{p}<0,001 * *)$, siendo las mujeres quienes la califican más frecuentemente como romántica y los hombres más como práctica y directa (Fig. 4).

Figura 4. Diferencias entre hombres y mujeres en la visión de la relación de pareja.

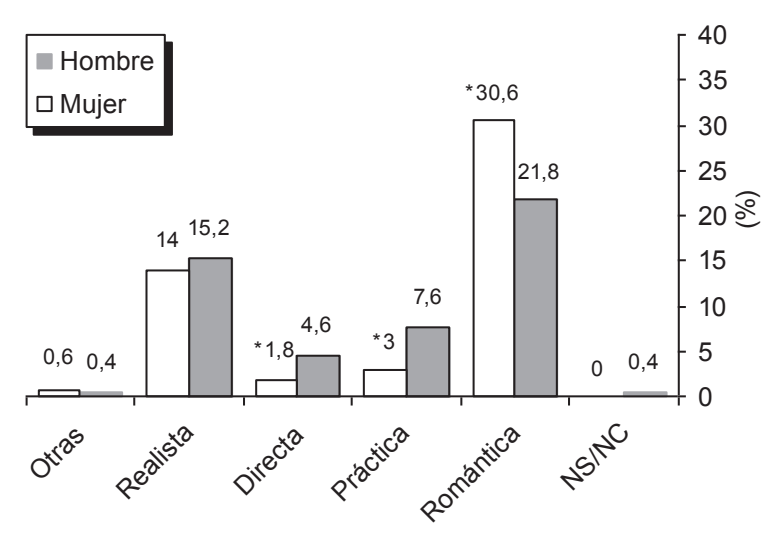

\section{Discusión}

\section{Estrategias de aproximación}

La formación de pareja se realiza en varios pasos cuya sucesión depende del conocimiento previo de ambos. El conocimiento que se tiene sobre cómo son dichas fases varía, tal y como se vislumbra a través de la encuesta realizada en una población de universitarios. Así, el 70\% reconoce que el inicio se produce mediante el contacto visual y que éste forma parte de las estrategias de aproximación. Son las mujeres quienes significativamente marcan esta pauta como inicio de la relación. Iniciar una posible relación con el contacto visual muestra a la otra parte su interés de manera sutil. Este contacto inicial también demuestra ser una táctica muy eficaz para las mujeres que intentan atraer a un compañero sexual. Los varones son muy sensibles a las señales que aumenten la probabilidad de sexo ocasional. En un estudio, se grabó en video a hombres y mujeres interactuando (Allan \& Fishell, 1979). Tras un breve tiempo, la mujer miraba al hombre a los ojos y le sonreía. Cuando otros hombres y mujeres veían la grabación juzgaron las intenciones de la mujer. Los hombres interpretaron esta conducta como un signo de interés sexual y un intento de seducción. Las mujeres interpretaron las mismas imágenes como un signo de amistad, sin connotaciones sexuales o seductoras. El 
contacto visual y la sonrisa son ambiguos, unas veces demuestran interés sexual y otras no, el contexto es el que ayuda a interpretar correctamente el sentido.

Durante el proceso de la seducción, las dos terceras partes de la muestra resaltan la necesidad de establecer una base de confianza y no se observan diferencias estadísticamente significativas entre las respuestas de ambos sexos. Establecer esta base de confianza implica mantener intercambios verbales para comprobar la disposición para ulteriores contactos. Es en esta fase donde se puede apreciar una mayor diversidad en función del marco cultural, que determina el dónde y el cómo es la estrategia de acercamiento.

Sobre las estrategias para encontrar intereses comunes, no se obtienen diferencias estadísticamente significativas entre hombres y mujeres. Más de la mitad prefiere encontrar los puntos en común relacionados con los rasgos de personalidad y una tercera parte resalta el compartir intereses socioculturales. En esta fase del festejo se ponen en común los intereses que se comparten y se expresan las coincidencias, aunque a veces la estrategia femenina pueda ser expresar opiniones contrarias para probar a la posible pareja. Lewis (1972) informa sobre lo que los norteamericanos de clase media especifican en este proceso, que comienzan por encontrar intereses comunes de tipo sociocultural, de valores, intereses y rasgos de personalidad. Kirkendall (1961) realizó una encuesta sobre las fases de galanteo a doscientos hombres en EEUU, cuyos resultados mostraban que para los hombres la presentación de uno mismo era muy importante en las primeras fases del contacto. Unos dan mucho valor a causar buena impresión, otros a exhibirse en compañía de sus amigas mostrando una estrategia más provocadora y por último están los que optan por solicitar pequeños favores para comprobar las buenas disposiciones de la posible pareja. Para establecer esta base de confianza, también se deben confiar a la pareja las debilidades aunque sin olvidar una presentación favorable de uno mismo.

\section{Posición de reclamo}

En la posición de reclamo el varón hace ostentación de que se halla en condiciones de dominar a otros. Esta posición está destinada a causar impresión, pero no se dirige contra la pareja. Las respuestas sobre las conductas que los universitarios emplean para mostrarse de forma favorable, "posición de reclamo", debían seleccionarlas y priorizarlas. Respecto a la ostentación de la capacidad de dominio sobre el entorno social, no se obtienen diferencias estadísticamente significativas entre los sexos, tampoco en la estrategia de utilizar "requerimientos infantiles" para provocar una respuesta protectora.

En cambio, sí se obtienen diferencias estadísticamente significativas entre hombres y mujeres respecto a la "presentación como un proveedor fiable", siendo una estrategia más valorada por las mujeres. Finalmente, el hombre y la mujer se presentan como una pareja apropiada para prestar cuidados, que responde al deseo de asistencia mutua y se logra mediante la transformación de requerimientos infantiles. En la muestra estudiada se observan diferencias estadísticamente significativas respecto a la estrategia de mantener una actitud juguetona, siendo los varones quienes valoran más esta conducta en las mujeres. También en las respuestas se observa que una tercera parte de la muestra marca como estrategia para mantener una posición de reclamo el provocar una respuesta protectora.

\section{Iniciativa al contacto}

Observaciones realizadas en diferentes culturas muestran que la iniciativa del contacto corporal suele ser del hombre, es él quien toca a su pareja de manera aparentemente casual (Eibl-Eibesfeldt, 1993). La mitad de los universitarios encuestados responde que son los varones quienes inician el contacto y se obtienen diferencias estadísticamente significativas en esta respuesta, reconociendo que son los hombres quienes realizan el primer contacto. El contexto en el que se produce este primer contacto es bailando y ayudando a la pareja.

Con los abrazos, caricias y besos se supera la barrera de la intimidad, éstas son las conductas que los universitarios seleccionan y priorizan para mostrar ternura. Primero son los besos, para más de la mitad de la población estudiada, seguidos de las caricias. No se obtienen diferencias estadísticamente significativas entre ambos sexos en las respuestas sobre estas muestras de cariño como introducción al acto sexual. En muchas culturas los contactos íntimos van precedidos por rituales formalizados de cortejo (Eibl-Eibesfeldt, 1971; Lewis, 1972; Pitcairn \& Schleidt, 1976).

\section{Sobre el festejo y la seducción}

En el inicio de la relación, una parte indica a la otra su interés y según la respuesta se produce, o no, una nueva aproximación. La mitad de la muestra estudiada opina que la fase del festejo más importante es la presentación de uno mismo y una tercera parte, el aparecer como deseable, independientemente del sexo. Cuando sí se observan diferencias en las respuestas entre sexos, es al preguntar sobre si la seducción es imprescindible. 
Las dos terceras partes de la muestra contestan que sí y son las mujeres las que contestan más afirmativamente.

En el estudio de Kirkendall (1962), sobre las fases de galanteo, los varones otorgaban gran importancia a la presentación de uno mismo en las primeras fases de contacto. Otra estrategia muy valorada consistía en que el hombre aparezca "deseable", exhibiéndose en compañía de sus amigas, pudiéndose interpretar como una estrategia provocadora y por último, pedir pequeños favores para comprobar la buena disposición de la pareja. Los estudios realizados por Buss (1988, 1994) y por Schmitt y Buss (2001), con universitarios sobre la atracción de la pareja, identificaron docenas de tácticas de atracción a partir de solicitarles que las identificaran y describieran, tanto las que observaban en ellos mismos como en otros. Estas eran: alardear de lo que se había conseguido, hablar de lo importantes que eran en el trabajo, mostrar comprensión por los problemas del otro, iniciar contacto visual y llevar ropa atractiva. Los investigadores redujeron el grupo de más de cien acciones a 28 categorías y solicitaron que 100 matrimonios y 200 universitarios evaluaran la eficacia de cada táctica, indicando si era más eficaz en una relación ocasional o en una estable y la frecuencia con la que ellos mismos las empleaban. Entre las tácticas masculinas valoradas como eficaces estaban las que consistían en exhibir recursos tangibles: demostrar que se gana mucho dinero, enseñarlo de forma ostentosa, alardear de lo que se ha conseguido para impresionar a las mujeres. Otra técnica masculina consiste en engañar sobre los recursos de que se dispone, mintiendo sobre las expectativas laborales y exagerando el prestigio. Por último, destacaban la de descalificar los recursos de los rivales para que parezcan menos atractivos al otro sexo.

La importancia de los recursos en la atracción no se limita a la cultura occidental, pues existen relatos de antropólogos que informan de cómo los cazadores con éxito tienen buen prestigio en la tribu y varias mujeres, incluso, le pueden abandonar por no tener éxito en la caza (Holmberg, 1950).

Tooke y Camire (1991) realizaron estudios sobre tácticas de atracción explotadoras y engañosas en una población universitaria, confeccionaron una lista de 88 formas en que los hombres y las mujeres se engañaban entre sí para atraer a una pareja. Los universitarios afirmaron que engañaban al otro sexo sobre las expectativas profesionales, escondían el estómago al pasar cerca de un miembro del sexo contrario, parecían más de fiar y más considerados de lo que realmente eran y actuaban como si realmente no les interesara el sexo cuando era en lo único que pensaban. Después, 252 universitarios evaluaron estas técnicas por su frecuencia y eficacia al usarlas un hombre o una mujer. En este estudio se halló que, para atraer a las mujeres, los hombres aparentan ser más corteses, considerados y vulnerables de lo que en realidad son.

\section{Visión sobre la relación de pareja}

Una tercera parte de los universitarios consultados considera que ha establecido una relación de pareja cuando se manifiesta una estimación mutua, y casi la mitad cuando también hay facilidad en la comunicación y después de mantener relaciones sexuales. Pero los hombres responden más frecuentemente que la relación se establece después de mantener relaciones sexuales y las mujeres cuando hay facilidad en la comunicación.

Respecto a lo que piensan sobre cómo evoluciona la relación de pareja, más de dos terceras partes $(88,3 \%)$ marca primero el establecimiento de confianza. En segundo lugar el tener un proyecto en común, y después la repartición de roles, la atención a los hijos y por último el interés patrimonial. Las diferencias entre hombres y mujeres son que ellas valoran más establecer un proyecto en común y el interés patrimonial. En esta visión de la evolución de la relación de pareja se pone de manifiesto cómo esta alianza complementa las habilidades, el hecho de compartir recursos, crear un entorno para criar a los hijos y al final establecer una red familiar más amplia.

Más de la mitad de los encuestados tiene una visión romántica de la pareja, un tercio la define como realista y menos del $10 \%$ como práctica. Las mujeres la califican más frecuentemente como romántica, en cambio los hombres de práctica y directa. Quizás estos datos ponen de manifiesto que la conducta de emparejamiento es muy flexible y sensible al contexto social. Para comprender las relaciones entre hombres y mujeres hay que fijarse tanto en las similitudes como en las diferencias.

Deseo, atracción y vínculo son tres emociones que se pueden estudiar a través de encuestas y que muestran cómo la evolución dio lugar a algunos comportamientos ahora universales que juegan a favor aunque no determinan. No obstante nos siguen atrayendo los rasgos físicos de nuestras parejas (Guillén-Salazar \& Pons-Salvador, 2002), a través de los caracteres sexuales secundarios, seguimos utilizando unas pautas de cortejo similares (Eibl-Eibesfeldt, 1993) al igual que las estrategias reproductivas (Buss \& Schmith, 1993). A pesar de que algunas de estas cuestiones requieren 
trabajos empíricos en poblaciones más amplias y representativas de las diferentes sociedades, estos estudios ilustran los cambios que se están produciendo en el comportamiento sexual humano.

\section{Referencias}

Allan, N. Fishell, D. (1979). Singles bars. En N. Allan (ed.). Urban life styles (pp128-179). Dubuque, IA: William C. Brown.

Buss, D. M. (1988). The evolution of human intrasexual competition. Journal of Personality and Social Psychology, 53, 1214-1221.

Buss, D. M. (1994). The evolution of Desire. Basic Books, Harper Collins Publishers.

Buss, D. M. \& Schmitt, D. P. (1993). Sexual strategies theory: An evolutionary perspective on human mating. Psychological Review, 100, 204-232.

Eibl-Eibesfeldt, I. (1971). Love and hate. The natural history of behaviour patterns. New York.

Eibl-Eibesfeldt, I.(1993). Biología del Comportamiento humano. Manual de etología humana. Madrid: Alianza Psicología.

Guillén-Salazar, F. \& Pons-Salvador, G. (2002). El origen evolutivo del comportamiento sexual humano: una aproximación desde el campo de la psicología evolucionista. Revista de Psicología General y Aplicada, 55 (2), 187-202.

Haberman S. J. (1978). Analysis of qualitative data (Vol I). New York: Academic.

Holmberg, A. R. (1950). Nomads of the long bow: The Siriono of Eastern Bolivia. Washington DC: US Govermment Printing Office.

Kirkendall, L. A. (1961). Premarital intercourse and interpersonal relationships. New York, Julien Press.
Lauman, E. O., Gagnon, J. H., Michale, R. T. \& Michaels, S. (1994). The social organization of sexuality. Sexual practices in the United States. The complete findings from America's most comprehensive survey of sexual behaviour. The University of Chicago Press. Chicago \& London.

Lewis, R. A. (1972). A developmental framework for the analysis of premarital dyadic formation. Family process, 11, 17-48.

Masters W. H. \& Jonson, V. E. (1966). Human sexual response. Boston, Little Brown \& Comp.

Maté, C. \& Acarín, N. (2010). Encuesta sobre las relaciones sexuales a los estudiantes de la Universitat Pompeu Fabra (20 a 27 años). Summa Psicológica UST, 7(2), 93-112.

Maté, C. \& Acarín, N. (2011). Encuesta sobre la elección de pareja a los estudiantes de la Universitat Pompeu Fabra (20 a 27 años). Summa Psicológica UST, 37-46.

Medina, E. A. (1991). El animal humano. Una introducción a su etología. Barcelona: Ed. Barcanova, Temas Universitarios.

Pitcairn, T. K. \& Schleidt, M. (1976). Dance and decision: An analysis of a courtship dance of the Medlpa, New Guinea. Behaviour, $58,298-316$.

Schmith, D. P. \& Buss, D. M. (2002). Human mate poaching: tactics and temptations for infiltrating existing relationships. Journal of personality and Social Psychology, 80, 894-917.

Tooke, J. \& Camire, L. (1991). Patterns of deception in intersexual and intrasexual mating strategies. Ethology and Sociobiology, $12,345-364$.

Wickler, W. \& Seibt, U. (1977). Song splitting in the evolution of dueting. Zeitschrift für Tierpsychologie, 59, 127-140.

Yasan, A., Essizoglu, A. \& Yildirim, E. A. (2009). Predictor factors associated with premarital sexual behaviour among university students in an Islamic culture. International Journal of Sexual Health, 21(3), 145-152. 